\title{
Single-incision laparoscopic surgery: an update of current evidence
}

\author{
Lok Yi Wu, Dominic Chi Chung Foo \\ Department of Surgery, The University of Hong Kong, Queen Mary Hospital, Hong Kong, China \\ Contributions: (I) Conception and design: DC Foo; (II) Administrative support: DC Foo; (III) Provision of study materials or patients: None; \\ (IV) Collection and assembly of data: None; (V) Data analysis and interpretation: None; (VI) Manuscript writing: All authors; (VII) Final approval of \\ manuscript: All authors. \\ Correspondence to: Dominic Chi Chung Foo. Department of Surgery, The University of Hong Kong, Queen Mary Hospital, 102 Pokfulam Road, \\ Hong Kong, China. Email: ccfoo@hku.hk.
}

\begin{abstract}
Single-incision laparoscopic surgery (SILS), especially for appendicectomy, colectomy, and cholecystectomy has attracted certain attention. They have the potential to improve patient satisfaction from "scar-free" cosmesis, decrease surgical trauma and reduce post-operative pain, and may hasten recovery. This article aims to review the current evidence regarding SILS for these three commonly indicated operations.
\end{abstract}

Keywords: Single-incision laparoscopic surgery (SILS); appendicectomy; colectomy; cholecystectomy

Received: 14 November 2016; Accepted: 16 November 2016; Published: 09 December 2016.

doi: $10.21037 /$ ales.2016.11.18

View this article at: http://dx.doi.org/10.21037/ales.2016.11.18

\section{Introduction}

Since the first recorded human operation dating back to the proto-neolithic times, surgical technique and technology have undergone tremendous transformations, in keeping with the undying medical tradition of pursuing excellence. With the advent of video-assisted laparoscopic surgery in the 1970's, various abdominal and gynecological surgeries have since adopted the laparoscopic approach, and many randomized controlled trials (RCTs) have proven their efficacy and benefits in terms of reducing surgical trauma, reducing the risk of surgical and medical complications, and faster return to daily activity when compared to open surgery. In order to further reduce surgical trauma, singleincision laparoscopic surgery (SILS), in which laparoscopic instruments are introduced through a single site, typically with a single incision at the umbilicus has been described.

It is imperative for each new technique to be vigorously studied in terms of feasibility and safety in comparison to the current standard treatment. This article would focus on the current evidence of three most widely performed and researched single-incision laparoscopic operations: single-incision laparoscopic appendicectomy (SILA), singleincision laparoscopic colectomy (SILC) and single-incision laparoscopic cholecystectomy (SILCh).

\section{SILA vs. conventional laparoscopic appendicectomy (CLA)}

A multitude of RCTs have been conducted to prove the feasibility and safety of SILA over CLA, many of which have good methodological quality. We examined several recently published meta-analyses and systematic reviews of these RCTs.

The largest meta-analyses to date is conducted by Xue et al. (1) in 2014, which included 11 RCTs with a collective total of 1,456 patients, roughly equally split into SILA and CLA groups. They found no difference in the primary outcomes of wound infection, intra-abdominal abscess, post-operative ileus and total postoperative complications. This finding was compatible with another meta-analysis of 8 RCTs by Xu et al. (2) in 2014 with a total of 1,234 patients, whose subgroup analysis of post-operative complications also includes wound and intra-abdominal infection, and paralytic ileus, and found that their rates were comparable between both groups. A meta-analysis of five RCTs by Vettoretto et al. (3), studying post-operative abdominal wall complications (defined as wound infection, bleeding, or hernia) found lower rates in the SILA group (4.9\% vs. 5.9\%), but these were not statistically significant.

Several authors mentioned one area in which SILA is 
superior to CLA with regards to operative morbidity. The requirement of inserting just one trocar in the umbilical area in SILA eliminates the possibility of injuring the bladder and the inferior epigastric vessels during trocar insertion in CLA. Injury to the epigastric vessels during CLA has been reported by two authors [Vettoretto et al. and Frutos et al. (4)], and reoperation was required in both cases. A meta-analysis by Chen et al. (5) studied reoperation rates in three studies. No patients required reoperation in the SILA group, whereas 2 patients in the CLA group underwent reoperation, one for appendiceal stump leakage, and the other for epigastric vessel injury. The odds ratio for reoperation was 0.33 (95\% CI, 0.03-3.19; $\mathrm{P}=0.34$ ) favoring SILA, but was not statistically significant.

Most systematic reviews demonstrated significant longer operative times for SILA compared to CLA. Xu et al.'s study showed a weighted mean difference of 5.96 minutes (95\% CI, 2.54-9.38; $\mathrm{P}=0.0006)$. Chen et al.'s study similarly showed a WMD of 5.38 minutes (95\% CI, 2.94-7.83; $\mathrm{P}<0.0001)$. Both results are of high heterogeneity, including trials in which surgeons are still in early phases of the learning curve, and hence should be interpreted with caution. Despite being statistically significant, a difference of five minutes is hardly clinically relevant. Moreover, several studies show that operative time can be shortened with experience (6), and Liao et al. (7) showed it is possible to achieve an operative time equivalent to that of SILA after the initial 30 cases.

Current understanding on the pathophysiology of post-operative wound pain is divided over two possible explanations. Those arguing for CLA being more painful suggest that post-surgical pain is mainly related to the injury of muscles and parietal peritoneum, and hence increased trauma in operations requiring more port sites. Yet those arguing for SILA being more painful suggest that the larger transumbilical fascial incision needed to accommodate surgical instruments results in greater wound irritation, compounded by its position in the umbilicus, where nerves are more sensitive than in the rest of the abdomen.

Empirically, analyses on post-operative pain were marred by the fact that different authors used different scales and evaluation times in their studies, resulting in great heterogeneity. RCTs by Frutos et al. and Kye et al. (8) noted statistically significant lower post-operative pain scores for SILA, in contrast to studies conducted by Teoh et al. (9), Sozutek et al. (10) and Lee et al., which did not show significant differences in pain scores. Perhaps a more objective measure of pain is the frequency and dosage of analgesia. Chen's meta-analysis studied two papers on the frequency of analgesic use and three papers on analgesic dosage. It showed no difference in the frequency of analgesia $(\mathrm{OR}=-0.13,95 \% \mathrm{CI},-0.44-0.18, \mathrm{P}=0.41)$, but the total dose of analgesia was significantly higher in the experimental group than in the control group (WMD $=0.96,95 \%$ CI, $0.45-1.47, \mathrm{P}=0.0002$ ).

In conclusion, most of the evidence to date showed that SILA and CLA were comparable in terms of safety and morbidity rates. Results of analyses on potential benefits, including less pain, better cosmesis, earlier return to activity and shorter hospital stay are still equivocal.

\section{SILC versus conventional multiport laparoscopic colectomy (CMLC)}

SILC for colon cancer was first described by Bucher et al. (11) and Remzi et al. (12) in 2008. We reviewed the only two RCTs available to date, conducted by Huscher et al. (13) and Poon et al. (14), as well as meta-analysis of comparative studies by Zhou et al. (15), Maggiori et al. (16) and Yang et al. (17).

The three meta-analyses demonstrated a comparable safety profile between SILC and CMLC. Yang et al.'s analysis of 13 studies showed a complication rate of $18.8 \%$ for SILC and $19.1 \%$ for CMLC, noting that the most common complications for both procedures were wound infection, paralytic ileus and anastomotic leakage. The pooled odds ratio was 0.83 (95\% $\mathrm{CI}, 0.57-1.20 ; \mathrm{P}=0.33$ ). This is echoed by Zhou et al.'s analysis of 12 studies, showing a complication rate of $22.1 \%$ in SILC and $23.4 \%$ in CMLC; $\mathrm{P}=0.28$. Moreover, Maggiori's meta-analysis of 15 studies showed no difference in post-operative morbidity between SILC and CMLC, with an odds ratio of 0.84 (95\% CI, 0.61-1.15; $\mathrm{P}=0.27$ ). Additionally, their study of 1,026 SILC in 64 studies showed that post-operative morbidity occurred in $17 \%$ of patients, with $1 \%$ requiring re-operation. The reasons included anastomotic leakage or bleeding, intra-abdominal bleeding, explorative laparoscopy for severe pain, wound hematoma and cecal ischemia with perforation.

Conversion rates to open laparotomy were also comparable between CMLC and SILC. Yang et al. noted a rate of $1.10 \%$ in SILC vs. $1.52 \%$ in CMLC, which was not statistically significant. Placement of an additional port was required in $6.44 \%$ of SILC vs. $1.01 \%$ in CMLC. Maggiori's meta-analysis also showed no difference in conversion rates to open between SILC and CMLC, odds ratio 0.58 (95\% 
CI, 0.24-1.38; $\mathrm{P}=0.22$ ). However, they noted significantly increased conversion rates for rectal surgery when compared with colonic resections. For single-incision rectal procedures, $25 \%$ required conversion to multiport and $3 \%$ to laparotomy. This is in contrast with single-incision colonic procedures in which conversion to multiport was required in $7 \%$ and conversion to laparotomy in $1 \%$.

Oncological clearance of single-incision approaches for colorectal cancer was also examined. Zhou's metaanalysis of 6 trials showed an equivalent number of lymph nodes harvested between the two groups (WMD $=1.71,95 \% \mathrm{CI},-0.41-3.83 ; \mathrm{P}=0.11)$. They also found no significant differences in lengths of proximal and distal margins. As for resection margins, they noted 4 out of 5 studies reporting clear pathologic resection margins in all patients who underwent SILC. The remaining study showed that for patients with positive margins, the rates were comparable between SILC and CMLC: $9.5 \%$ vs. $11.3 \%$ respectively, $\mathrm{P}=0.8$. On the other hand, Yang et al. noted that in their analysis of 8 studies, the number of harvested lymph nodes was significantly more for SILC, pooled $\mathrm{WMD}=1.75$ (95\% CI, 0.12-3.38; $\mathrm{P}=0.035$ ).

Papaconstantinou et al. (18) conducted a case-matched comparative study with a mean follow-up of 13 months, and found an identical disease-free survival rate of $92 \%$ for both groups, $\mathrm{P}=0.97$. Within the follow-up period, there were no reported port-site recurrences in both groups. This is supported by Huscher et al.'s RCT with a follow-up of 22 months, also showing no port-site recurrences. Yun et al.'s retrospective analysis (19) also show a comparative disease-free survival, with $89.7 \%$ for SILC and $96.3 \%$ for CMLC, $\mathrm{P}=0.120$. There was also no statistically significant difference in recurrence rate, $9.1 \%$ for SILC and $3.2 \%$ for CMLC, $\mathrm{P}=0.120$.

Current data on post-operative pain based on retrospective studies are mixed at best. At worst they are inadequate for assessing this outcome, limited by failure of homogeneous comparison and inherent bias due to study design. Only one RCT has examined this topic: Poon et al.'s study in 2012 included 50 patients with similar pathology requiring colectomy, with 25 patients randomized into each arm, similar demographic characteristics, using a standardized anesthetic protocol and blinded patients and research staff (14). They reported lower median wound pain score at rest for SILC group than in the CMLC group, with statistically significant differences in post-op day 1 and 2 . They also found that patients with SILC had a shorter median hospital stay, 4 vs. 5 days; $\mathrm{P}<0.001$.
The three meta-analyses also unanimously demonstrated that SILC is associated with significantly shorter duration of hospital stay. Maggiori et al. showed WMD of -0.75 (95\% CI, -1.30 to $-0.20 ; \mathrm{P}=0.008)$, Yang et al. showed WMD of -0.68 (95\% CI, -1.20 to $-0.16 ; \mathrm{P}=0.0099)$ and $\mathrm{Zhou}$ et al. showed WMD of -0.32 (95\% CI, -0.52 to -0.12 ; $\mathrm{P}=0.002$ ). Reduced post-operative pain, as demonstrated by Poon et al., may be a possible explanation. Furthermore, in view of comparable complication profile, another possible explanation is faster postoperative recovery with SILC. Several studies show earlier return of bowel movement and resumption of normal diet, and Zhou's meta-analysis showed that in SILC, time to first flatus was significantly shorter, $W M D=-0.58$ (95\% CI, -0.85 to $-0.30 ; \mathrm{P}<0.001)$.

In conclusion, the current evidence suggests that the single-incision approach is a feasible alternative to conventional laparoscopic techniques, with comparable complication rates and conversion rate to open laparotomy. Adherence to oncological principles is also proven with meta-analyses showing similar number of lymph nodes harvested, length of resection margins and rate of $\mathrm{R} 0$ resection. Long-term oncological outcomes are currently unable to be assessed given the lack of long-term follow up from the available studies. Evidence also suggested a potential advantage of less post-operative pain and shorter hospital stay.

\section{SILCh versus conventional laparoscopic cholecystectomy (CLC)}

SILCh was first described in 1997 (20). Due to its earlier introduction in contrast to single-incision appendicectomy and colectomy, specific outcomes like post-operative pain, cosmetic satisfaction, and complication rates have been extensively studied.

The most updated systematic review of available RCTs is conducted by Lirici et al. (21) in 2016, including 17 RCTs. The study by Milas et al. (22) in 2014 included the largest number of RCTs among all other systematic reviews to date [30]. We mainly discuss the outcomes detailed in these papers.

Fifteen of 17 RCTs in Lirici et al.'s review reported on post-operative pain, and all used the visual analogue scale (VAS), although different time intervals were used. They found that post-operative pain was significantly worse for CLC in 4 studies, while 4 other studies show worse pain for SILCh patients instead, and the remaining 7 studies showed no difference in pain scores. Twenty-two of 30 
studies in Milas et al.'s systematic review reported on postoperative pain, but used different measurement tools. Thus they conducted random-effects meta-analysis at $24 \mathrm{~h}$ postsurgery, and found that the difference tended towards statistical significance in favor of SILCh with less pain (SMD $-0.30, \mathrm{P}=0.095)$.

With regards to complications, common ones were bile duct injuries, bile leak, biliary collection or abscess, retained stones, wound complications like infection, and postoperative incisional hernia. Lirici et al. demonstrated similar morbidity rates for SILCh and CLC groups, although not statistically significant. Milas et al., by using the bivariate binomial-normal (BN) method (designed for sparse dichotomous data i.e. no continuity correction, inclusion of all trials), demonstrated that the estimated random-effects incidence of complications was $5.35 \%(103 / 1,209)$ for SILCh and $3.79 \%(79 / 1,202)$ for CLC, although with high between-trial heterogeneity. They also specifically studied the occurrence of incisional hernia. By using the unweighted method by Shuster, they showed a higher risk of incisional hernia with SILC $(\mathrm{OR}=4.94, \mathrm{P}=0.025)$, although the total number of events was low (15/839 SILCh, 4/837 CLC).

This sheds light on operative outcome in view of the fact that previous systematic reviews have not been able to demonstrate statistically significant differences in the incidence or risk of post-operative complications or incisional hernias. For example, Garg et al.'s study (23) of 9 RCTs in 2012 showed higher incidence of complications in SILCh, $16 \%$ vs. $12.3 \%$ in CLC (RR $=1.21,95 \%$ CI, $0.73-$ 2.01; $\mathrm{P}=0.45$ ), and also higher incidence of hernia in SILCh, $1.43 \%$, vs. $0.32 \%$ in CLC ( $R R=2.94,95 \%$ CI, $0.47-18.33$; $\mathrm{P}=0.25)$. Similarly, Trastulli et al.'s study (24) of 13 RCTs showed that the pooled odds ratio for complications was 1.14 in favor of CLC $(\mathrm{P}=0.61)$, and that for incisional hernia was 2.99 in favor of CLC $(\mathrm{P}=0.18)$. Yet none of their results were statistically significant.

Previously we have touched on the effect of learning curve on operating time, noting that many studies were limited by the fact that they were conducted during the early phases of a surgeon's training in SILS. Hence it was difficult to attribute measured time differences as being specific to surgical skills, which is amenable to training, or inherent to the approach. Milas et al. are the only authors to provide closure in this regard. With conventional analytic methods not accounting for the learning curve, operating time was 12.4 min longer in SILCh $(\mathrm{P}<0.001)$, understandably with high heterogeneity among trials. Through metaregression analysis accounting for differential expertise bias, heterogeneity was resolved, by isolating studies in which the surgeons had pre-trial SILCh experience or $>40$ operations in the SILCh arm i.e., with a low risk of expertise bias. In analysis for studies with low risk of bias, the weighted mean difference of operating time between SILCh and MLC was 9.6 minutes $(\mathrm{P}=0.40)$, whereas the WMD calculated using studies with high risk of bias was 21.1 minutes. The effect was more dramatic when looking at trials with $n>40$ in the SILCh arm, WMD being 5.9 vs. 16.8 minutes for trials with only 25 patients or less in the SILCh arm. This shows that more experience with SILCh is indeed associated with shorter operating times.

In their study they also demonstrated similar conclusions on procedure failure rate, for SILCh defined as addition of a trocar, or conversion to CLC or open surgery, whereas for CLC it is defined as addition of a trocar or conversion to open surgery. The main reasons for converting SILCh include limited visualization of Calot's triangle, arterial bleeding, and difficulty in dissection of ducts (21). When not accounting for heterogeneity, the pooled incidence of failure with SILCh is $4.39 \%$ vs. $0.53 \%$ for CLC ( $\mathrm{P}=0.019)$. When looking at SILCh alone, the risk of procedure failure in 10 trials with low risk of expertise bias is $3.60 \%$ vs. $5.16 \%$ in trials with high or uncertain risk of bias. This once again shows that experience with SILCh is associated with reduced procedure failure rate.

As for cosmetic satisfaction, most systematic reviews were unanimously in favor of SILCh. Milas et al. demonstrated in an analysis of 16 trials that satisfaction with SILCh is greater than that for CLC at all-time points post-op. The largest difference was at $1-3$ months post-surgery, with a standard mean difference of $0.99(\mathrm{P}<0.001)$. Lirici et al. also reported that in their study of 17 trials, all but two showed significantly better cosmetic results for SILCh than for CLC. Garg et al. also showed a WMD of 1.00 (95\% CI, 0.49-1.51; $\mathrm{P}=0.0001)$, significantly better in SILCh than for CLC. However, all studies noted that data for long term post-op follow up were still lacking, which was problematic since they might not cover periods when incisional hernias start to arise and when the peripheral scars in CLC start to improve.

In conclusion, most systematic reviews demonstrate a slightly higher incidence of complications in SILCh, and especially with a higher risk for port-site hernia, although the total number was low. Only Milas et al.'s study was able to show a statistically significant difference. Debate still exists regarding the benefit of SILCh in reducing postoperative pain. Benefit in terms of cosmesis was clear from 
all trials from the analysis of mid-term data.

Apart from conventional and single-incision approaches, many other variations of laparoscopic cholecystectomy, including 2-port, 3-port, mini-laparoscopic and robotassisted cholecystectomy are undergoing comparative evaluation. Li et al.'s network meta-analysis (25) of 43 RCTs provided extensive discussion on the individual benefits of each approach. Moreover, even within the singleincision approach, various new techniques, maneuvers and instruments were being tested in different centers worldwide, promising exciting times ahead.

\section{Conclusions}

SILS is applicable to a number of procedures with cholecystectomy, appendicectomy and colectomy being the three most widely described and researched. The current literature demonstrated their feasibility and safety in terms of comparable overall complication rates and oncological clearance in cancer surgery. There was a concern of higher incidence of certain complication like port site hernia, although the overall incidence was low. The advantage of improved patient satisfaction due to better cosmesis has been proven by many. Some studies also demonstrated a potential for less postoperative pain and faster recovery but evidence on this is still inconclusive. Future studies would benefit from standardization of pain and methods of assessment.

\section{Acknowledgements}

None.

\section{Footnote}

Conflicts of Interest: The authors have no conflicts of interest to declare.

\section{References}

1. Xue C, Lin B, Huang Z, et al. Single-incision laparoscopic appendectomy versus conventional 3-port laparoscopic appendectomy for appendicitis: an updated metaanalysis of randomized controlled trials. Surg Today 2015;45:1179-86.

2. Xu AM, Huang L, Li TJ. Single-incision versus threeport laparoscopic appendectomy for acute appendicitis: systematic review and meta-analysis of randomized controlled trials. Surg Endosc 2015;29:822-43.

3. Vettoretto N, Cirocchi R, Randolph J, et al. Acute appendicitis can be treated with single-incision laparoscopy: a systematic review of randomized controlled trials. Colorectal Dis 2015;17:281-9.

4. Frutos MD, Abrisqueta J, Lujan J, et al. Randomized prospective study to compare laparoscopic appendectomy versus umbilical single-incision appendectomy. Ann Surg 2013;257:413-8.

5. Chen JM, Geng W, Xie SX, et al. Single-incision versus conventional three-port laparoscopic appendectomy: A meta-analysis of randomized controlled trials. Minim Invasive Ther Allied Technol 2015;24:195-203.

6. Lee JS, Choi YI, Lim SH, et al. Transumbilical single port laparoscopic appendectomy using basic equipment: a comparison with the three ports method. J Korean Surg Soc 2012;83:212-7.

7. Liao YT, Lin TH, Lee PC, et al. Learning curve of singleport laparoscopic appendectomy for noncomplicated acute appendicitis: a preliminary analysis compared with conventional laparoscopic appendectomy. J Laparoendosc Adv Surg Tech A 2013;23:441-6.

8. Kye BH, Lee J, Kim W, et al. Comparative study between single-incision and three-port laparoscopic appendectomy: a prospective randomized trial. J Laparoendosc Adv Surg Tech A 2013;23:431-6.

9. Teoh AY, Chiu PW, Wong TC, et al. A double-blinded randomized controlled trial of laparoendoscopic single-site access versus conventional 3-port appendectomy. Ann Surg 2012;256:909-14.

10. Sozutek A, Colak T, Dirlik M, et al. A prospective randomized comparison of single-port laparoscopic procedure with open and standard 3-port laparoscopic procedures in the treatment of acute appendicitis. Surg Laparosc Endosc Percutan Tech 2013;23:74-8.

11. Bucher P, Pugin F, Morel P. Single port access laparoscopic right hemicolectomy. Int J Colorectal Dis 2008;23:1013-6.

12. Remzi FH, Kirat HT, Kaouk JH, et al. Single-port laparoscopy in colorectal surgery. Colorectal Dis 2008;10:823-6.

13. Huscher CG, Mingoli A, Sgarzini G, et al. Standard laparoscopic versus single-incision laparoscopic colectomy for cancer: early results of a randomized prospective study. Am J Surg 2012;204:115-20.

14. Poon JT, Cheung CW, Fan JK, et al. Single-incision versus conventional laparoscopic colectomy for colonic neoplasm: a randomized, controlled trial. Surg Endosc 2012;26:2729-34. 
15. Zhou YM, Wu LP, Zhao YF, et al. Single-incision versus conventional laparoscopy for colorectal disease: a metaanalysis. Dig Dis Sci 2012;57:2103-12.

16. Maggiori L, Gaujoux S, Tribillon E, et al. Single-incision laparoscopy for colorectal resection: a systematic review and meta-analysis of more than a thousand procedures. Colorectal Dis 2012;14:e643-54.

17. Yang TX, Chua TC. Single-incision laparoscopic colectomy versus conventional multiport laparoscopic colectomy: a meta-analysis of comparative studies. Int J Colorectal Dis 2013;28:89-101.

18. Papaconstantinou HT, Thomas JS. Single-incision laparoscopic colectomy for cancer: assessment of oncologic resection and short-term outcomes in a casematched comparison with standard laparoscopy. Surgery 2011;150:820-7.

19. Yun JA, Yun SH, Park YA, et al. Single-incision laparoscopic right colectomy compared with conventional laparoscopy for malignancy: assessment of perioperative and short-term oncologic outcomes. Surg Endosc 2013;27:2122-30.

doi: 10.21037/ales.2016.11.18

Cite this article as: Wu LY, Foo DC. Single-incision laparoscopic surgery: an update of current evidence. Ann Laparosc Endosc Surg 2016;1:45.
20. Navarra G, Pozza E, Occhionorelli S, et al. One-wound laparoscopic cholecystectomy. Br J Surg 1997;84:695.

21. Lirici MM, Tierno SM, Ponzano C. Single-incision laparoscopic cholecystectomy: does it work? A systematic review. Surg Endosc 2016;30:4389-99.

22. Milas M, Deveđija S, Trkulja V. Single incision versus standard multiport laparoscopic cholecystectomy: up-dated systematic review and meta-analysis of randomized trials. Surgeon 2014;12:271-89.

23. Garg P, Thakur JD, Garg M, et al. Single-incision laparoscopic cholecystectomy vs. conventional laparoscopic cholecystectomy: a meta-analysis of randomized controlled trials. J Gastrointest Surg 2012;16:1618-28.

24. Trastulli S, Cirocchi R, Desiderio J, et al. Systematic review and meta-analysis of randomized clinical trials comparing single-incision versus conventional laparoscopic cholecystectomy. Br J Surg 2013;100:191-208.

25. Li L, Tian J, Tian H, et al. The efficacy and safety of different kinds of laparoscopic cholecystectomy: a network meta analysis of 43 randomized controlled trials. PLoS One 2014;9:e90313. 